\title{
A preference foundation for constant loss aversion
}

Citation for published version (APA):

Peters, H. J. M. (2010). A preference foundation for constant loss aversion. METEOR, Maastricht University School of Business and Economics. METEOR Research Memorandum No. 062 https://doi.org/10.26481/umamet.2010062

Document status and date:

Published: 01/01/2010

DOI:

10.26481/umamet.2010062

Document Version:

Publisher's PDF, also known as Version of record

\section{Please check the document version of this publication:}

- A submitted manuscript is the version of the article upon submission and before peer-review. There can be important differences between the submitted version and the official published version of record.

People interested in the research are advised to contact the author for the final version of the publication, or visit the DOI to the publisher's website.

- The final author version and the galley proof are versions of the publication after peer review.

- The final published version features the final layout of the paper including the volume, issue and page numbers.

Link to publication

\footnotetext{
General rights rights.

- You may freely distribute the URL identifying the publication in the public portal. please follow below link for the End User Agreement:

www.umlib.nl/taverne-license

Take down policy

If you believe that this document breaches copyright please contact us at:

repository@maastrichtuniversity.nl

providing details and we will investigate your claim.
}

Copyright and moral rights for the publications made accessible in the public portal are retained by the authors and/or other copyright owners and it is a condition of accessing publications that users recognise and abide by the legal requirements associated with these

- Users may download and print one copy of any publication from the public portal for the purpose of private study or research.

- You may not further distribute the material or use it for any profit-making activity or commercial gain

If the publication is distributed under the terms of Article $25 \mathrm{fa}$ of the Dutch Copyright Act, indicated by the "Taverne" license above, 


\section{Maastricht University}

Hans Peters

A preference foundation for constant loss aversion

RM/ 10/062

\section{METEOR}

Maastricht University School of Business and Economics

Maastricht Research School of Economics

of Technology and Organization

P.O. Box 616

NL - 6200 MD Maastricht

The Netherlands 


\title{
A preference foundation for constant loss aversion*
}

\author{
Hans Peters ${ }^{\dagger}$
}

November 2010

\begin{abstract}
Following prospect theory we consider decision making under risk in which the decision maker's preferences depend on a reference outcome. An outcome below this reference outcome is regarded as resulting from a loss: a loss decreases the decision maker's basic utility more than a comparable gain increases this utility. An elegant and simple method to model this phenomenon was proposed by Shalev (2002): the utility of an outcome below the reference outcome is obtained from the basic utility by subtracting a multiple of the loss in basic utility: this multiple, the loss aversion coefficient, is constant across different reference outcomes. We provide a preference foundation for this loss aversion model.
\end{abstract}

JEL-codes: D81, C60

Keywords: Decision Making under Risk, Reference Outcome, Loss Aversion

\section{Constant loss aversion}

The concept of loss aversion has received wide attention in the theoretical, experimental and empirical psychological and economic literature over the past decades. Loss aversion is an important ingredient of prospect theory (Kahneman and Tversky, 1979). Kahneman (2003, p. 726) writes: "The concept of loss aversion was, I believe our [Tversky's and Kahneman's] most useful contribution to the study of decision making." According to prospect theory, a decision maker's preference is characterized by a basic utility function, a reference outcome, and a pair of probability weighting functions. Outcomes below the reference outcome are experienced as resulting from losses, and their utilities are decreased relative to the basic utilities. Probabilities in lotteries are transformed by weighting functions, again possibly depending on whether they involve losses or gains.

The present paper focuses on loss aversion while ignoring the probability weighting effect. This is partly for convenience and partly since indeed loss

\footnotetext{
${ }^{*}$ Thanks are due to Peter Wakker for many helpful comments. The usual disclaimer applies.

${ }^{\dagger}$ Department of Quantitative Economics, Maastricht University, P.O. Box 616, 6200 MD Maastricht, The Netherlands. Email: h.peters@maastrichtuniversity.nl.
} 


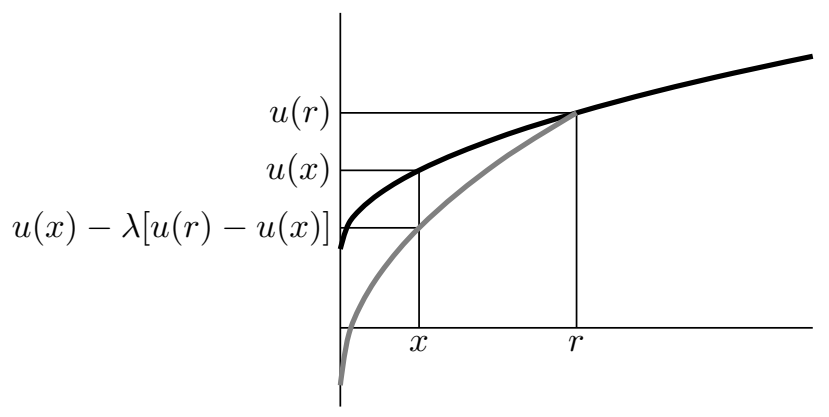

Figure 1. An illustration of (1). The black curve is the graph of the function $u$. Below $r$ the gray curve is the graph of $u^{r, \lambda}$, above $r$ this function coincides with $u$. (In this picture we took $u(x)=\sqrt{x}+1$ for $x \geqslant 0$ and $\lambda=1$.)

aversion can be separated from probability weighting and even from risk (e.g., Tversky and Kahneman, 1991; Köbberling and Wakker, 2005). ${ }^{1}$ The central assumption of loss aversion is that the decrease in utility resulting from a loss relative to the reference outcome is larger than the increase in utility resulting from a comparable gain. For example, the decrease in utility of losing one Euro if one has or expects to have 10 Euros is larger than the increase in utility of gaining one Euro if one has or expects to have 9 Euros. The literature, however, proposes quite different ways to measure this. ${ }^{2}$ For instance, Tversky and Kahneman (1992) take the ratio of the utility of a loss of one monetary unit (relative to reference level zero) and the utility of a gain of one unit as an index of loss aversion. Köbberling and Wakker (2005) take the ratio of the left derivative and the right derivative of the utility function at reference outcome zero.

The latter approach is consistent with the model of loss aversion proposed by Shalev (2002). In this model, utilities below the reference outcome are scaled down by subtracting the losses multiplied by a constant factor $\lambda$, called the loss aversion coefficient. For example (cf. Figure 1), let the nondecreasing function $u: A \rightarrow \mathbb{R}$, where $A \subseteq \mathbb{R}$, be a basic utility function, let $r \in A$ be a reference outcome, and let $\lambda \geqslant 0$ be the loss aversion coefficient. The utility function $u^{r, \lambda}$, which takes loss aversion into account, is defined by

$$
u^{r, \lambda}(x)= \begin{cases}u(x) & \text { if } x \in A, x \geqslant r \\ u(x)-\lambda[u(r)-u(x)] & \text { if } x \in A, x<r .\end{cases}
$$

Then a decision maker with basic utility function $u$ and reference outcome $r$ evaluates risky outcomes (lotteries) by computing the expected utility using $u^{r, \lambda}{ }^{3}$

\footnotetext{
${ }^{1}$ Many applications consider loss aversion without probability weighting, e.g., Dittman et al. (2009), Driesen et al. (2010), Dunn (1996), Freund and Özden (2008), Genesove and Mayer (2001), Götte et al. (2004), and Rosenblatt-Wisch (2008). Also Sugden (2003) does not consider probability weighting.

${ }^{2}$ An overview of several methods is given in Abdellaoui et al. (2007).

${ }^{3}$ In the literature, $r$ is often fixed and taken to be 0 , with $u(0)=0$. In that case, the utility
} 
Shalev's model is particularly simple and elegant, and easy to apply due to its basic assumption of a constant loss aversion parameter. The word 'constant' refers to two different aspects of this way of modelling loss aversion. First, for a given reference outcome the same multiple $\lambda$ of the loss is subtracted from the basic utility values for different outcomes $x$. Second, this multiple is constant across different reference outcomes, i.e., does not depend on $r$. The first aspect is quite common in applications, which in particular implies that many applications are consistent with the way Shalev models loss aversion; the second aspect of course only possibly plays a role in applications if the reference outcome varies. ${ }^{4}$

In this paper we present a preference foundation for (1) in a slightly more general model, that is, for a more general set of outcomes instead of $\mathbb{R}$. Like Sugden (2003) or Schmidt (2003), we assume that a decision maker has 'triadic' preferences: his preference over any two outcomes depends on a third (reference) outcome. We consider decision making under risk, and basically assume that for any given reference outcome the decision maker is an expected utility maximizer whose ordinal ranking of riskless outcomes does not depend on the reference outcome. Our main conditions focus on the consequences of changing reference outcomes.

Somewhat deviating from other characterizations of loss aversion (Sugden, 2003, or Schmidt, 2003) we start by assuming expected utility maximization for any fixed reference point, and focus on conditions that characterize constant loss aversion as in (1). Unlike Sugden (2003) or Köszegi and Rabin (2006) we assume that the reference outcome is riskless: we will comment on this assumption in Section 3, where we argue that it does not imply much loss of generality. Köbberling and Wakker (2005) characterize a specific form of constant loss aversion in a model without risk and with a fixed reference outcome, and focus on comparison of loss aversion between different decision makers.

The next section presents the model and characterization of constant loss aversion, and Section 3 contains further discussion.

\section{The preference model and characterization}

Let $A$ be a set of (riskless) outcomes. To avoid trivial cases we assume that $A$ has at least three elements. Let $\mathcal{L}$ denote the set of lotteries over $A$, i.e., the set of probability distributions over $A$ with finite support. We identify each $a \in A$ with the lottery that puts probability 1 on $a$.

A binary relation $R$ on some set $X$ is a weak ordering if it is complete, i.e., $(x, y) \in R$ or $(y, x) \in R$ for all $x, y \in X$, and transitive.

Let $R$ be a binary relation on $A$, and for each $a \in A$ let $\succcurlyeq^{a}$ be a binary relation on $\mathcal{L}$. The relation $R$ is interpreted as a decision maker's ranking of

of $x<0$ is equal to $(1+\lambda) u(x)$; then $\mu=1+\lambda$ is called the loss aversion coefficient and $\mu \geqslant 1$. See, e.g., Köbberling and Wakker (2005).

${ }^{4}$ See for instance Dittman et al. (2009). Some of the other applications mentioned in footnote 1 impose, indeed, a constant loss aversion parameter for a fixed reference outcome. 
the riskless outcomes, whereas $\succcurlyeq^{a}$ is his preference over lotteries if the reference outcome is $a$.

For $a, b \in A$ and $\ell, \ell^{\prime} \in \mathcal{L}$ we write $a R b$ instead of $(a, b) \in R$ and $\ell \succcurlyeq^{a} \ell^{\prime}$ instead of $\left(\ell, \ell^{\prime}\right) \in \succcurlyeq^{a}$. By $P$ we denote the asymmetric part of $R$. By $\operatorname{supp}(\ell) \subseteq$ $A$ we denote the support of $\ell \in \mathcal{L}$. For $x \in A, \ell(x)$ is the probability assigned by $\ell$ to $x$, and for $B \subseteq A, \ell(B)=\sum_{x \in B} \ell(x)$. We write $\succcurlyeq$ for the collection $\left\{\succcurlyeq^{a} \mid a \in A\right\}$.

We will assume that $R$ and all $\succcurlyeq^{a}$ are weak orderings. Besides this assumption we consider five conditions on the pair $(R, \succcurlyeq)$. The first two conditions set the general stage and are not directly related to loss aversion. The first condition says that each $\succcurlyeq^{a}$ coincides with $R$ when only riskless outcomes are concerned.

Common Ordinal Ranking (COR): For all $a, x, y \in A, x \succcurlyeq^{a} y \Leftrightarrow x R y$.

The second condition states that each preference $\succcurlyeq^{a}$ can be represented by an expected utility function, which is unique up to positive affine transformations. This holds under standard conditions, such as independence and continuity (e.g., Herstein and Milnor, 1953). For a function $u: A \rightarrow \mathbb{R}$ we denote by $E u(\ell)=\sum_{x \in A} \ell(x) u(x)$ the expected utility of $\ell \in \mathcal{L}$ under $u$.

Expected Utility (EU): For all $a \in A$ there is a function $u^{a}: A \rightarrow \mathbb{R}$ such that $\ell \succcurlyeq^{a} \ell^{\prime} \Leftrightarrow E u^{a}(\ell) \geqslant E u^{a}\left(\ell^{\prime}\right)$ for all $\ell, \ell^{\prime} \in \mathcal{L}$.

The next two conditions concern loss aversion but both describe situations in which preferences should not depend on reference outcomes. The first one states that if two lotteries both involve only losses relative to reference outcome $a$ and only gains relative to reference outcome $b$, then the preference between these two lotteries does not depend on whether $a$ or $b$ is the reference outcome. This is a special instance of the general principle that reference outcomes only influence preferences between gains and losses.

Reference Outcome Independence-1 (ROI-1): For all $a, b \in A$ with $a R b$ and all $\ell, \ell^{\prime} \in \mathcal{L}$, if $a R x R b$ for all $x \in \operatorname{supp}(\ell) \cup \operatorname{supp}\left(\ell^{\prime}\right)$, then $\ell \succcurlyeq^{a} \ell^{\prime} \Leftrightarrow \ell \succcurlyeq^{b} \ell^{\prime}$.

The second of these two independence conditions says the following. Consider two different lotteries and a reference outcome such that the total weight on outcomes involving losses is equal in both lotteries. Then a change in reference point without changing losses into gains or vice versa in either lottery, does not change the preference between these two lotteries. In contrast with the previous condition ROI-1, this condition concerns lotteries involving both gains and losses. As will become apparent in the proof of the characterization result, it guarantees that the loss aversion coefficient is a constant and does not depend on the reference outcome. 
Reference Outcome Independence-2 (ROI-2): For all $a, b \in A$ with $a R b$ and all $\ell, \ell^{\prime} \in \mathcal{L}$, if (i) $\left[\operatorname{supp}(\ell) \cup \operatorname{supp}\left(\ell^{\prime}\right)\right] \cap\{x \in A \mid a P x P b\}=\emptyset$ and (ii) $\ell(\{x \in A \mid$ $b R x\})=\ell^{\prime}(\{x \in A \mid b R x\})$, then $\ell \succcurlyeq^{a} \ell^{\prime} \Leftrightarrow \ell \succcurlyeq^{b} \ell^{\prime}$.

ROI-2 implies as special cases that preferences between two lotteries are independent of reference outcomes as long as both lotteries involve only gains or only losses relative to these reference outcomes, that is, if $a R b$ and either $x R a$ for all $x \in \operatorname{supp}(\ell) \cup \operatorname{supp}\left(\ell^{\prime}\right)$ or $b R x$ for all $x \in \operatorname{supp}(\ell) \cup \operatorname{supp}\left(\ell^{\prime}\right)$.

The final condition captures a basic intuition behind loss aversion, namely that a lower reference outcome can only increase preference. Suppose a loss averse decision maker prefers a lottery $\ell$ over outcome $a$, and $a$ is also his reference outcome. Then, if his reference outcome worsens from $a$ to $b$, he still prefers $\ell$ over $a$ under the new reference outcome $b$. To elaborate on this intuition, note that outcomes of $\ell$ representing gains relative to $a$ still represent gains with respect to $b$ : such outcomes should not lead to a change in preference. Outcomes of $\ell$ representing losses with respect to $b$ represent even more severe losses with respect to $a$ and, thus, this should only reinforce the preference of $\ell$ over $a$ when $b$ becomes the reference outcome. Outcomes of $\ell$ that represent losses relative to $a$ but gains relative to $b$ should certainly only reinforce the preference of $\ell$ over $a$ when $b$ becomes the reference outcome.

Reference Outcome Dependence (ROD): For all $a, b \in A$ and $\ell \in \mathcal{L}$, if $a R b$ then $\ell \succcurlyeq^{a} a \Rightarrow \ell \succcurlyeq^{b} a$.

Further discussion on these conditions is postponed until Section 3.

Let $u: A \rightarrow \mathbb{R}$ be a function satisfying $u(x) \geqslant u(y) \Leftrightarrow x R y$ for all $x, y \in A$. Let $\lambda \geqslant 0$, and let $a \in A$. Similar to (1) we define for all $x \in A$

$$
u^{a, \lambda}(x)= \begin{cases}u(x) & \text { if } x R a \\ u(x)-\lambda[u(a)-u(x)] & \text { if } a P x .\end{cases}
$$

For a lottery $\ell \in \mathcal{L}$, we denote by

$$
E u^{a, \lambda}(\ell)=\sum_{x \in A} \ell(x) u^{a, \lambda}(x)
$$

the expected utility of $\ell$ with reference point $a$. We can now state our characterization result. Its proof is in the Appendix.

Theorem 1 For the pair $(R, \succcurlyeq)$ the following two statements are equivalent.

(i) $R$ and $\succcurlyeq^{a}$ are weak orderings for all $a \in A$, and $(R, \succcurlyeq)$ satisfies COR, EU, ROI-1, ROI-2, and ROD.

(ii) There exists a function $u: A \rightarrow \mathbb{R}$ and a real number $\lambda \geqslant 0$ such that

(ii.1) $u(x) \geqslant u(y) \Leftrightarrow x R y$ for all $x, y \in A$.

(ii.2) $\ell \succcurlyeq^{a} \ell^{\prime} \Leftrightarrow E u^{a, \lambda}(\ell) \geqslant E u^{a, \lambda}\left(\ell^{\prime}\right)$ for all $a \in A$ and $\ell, \ell^{\prime} \in \mathcal{L}$.

Moreover, if (ii) holds for another function $v$, then there are $\alpha, \beta \in \mathbb{R}$ with $\alpha>0$ such that $v(x)=\alpha u(x)+\beta$ for all $x \in A$. 


\section{Further discussion}

Our basic framework is set by the conditions COR and EU together with the assumption of weak ordering. We have already commented on the absence of probability weighting. As to the assumption of weak ordering one could make a case for allowing incompleteness. Bleichrodt (2007) argues that if the reference outcome is an available option (e.g., keeping your present job) then a decision maker may not have a preference between two other outcomes (e.g., two other jobs that he dislikes compared to his present job) if he never has to choose between those jobs, e.g., since he prefers to keep his current job. This is a valid point, which calls for a revealed preference approach; this is not pursued here.

Remark 1 It is possible to drop condition COR, pick an arbitrary $a \in A$, and define $R$ by $x R y: \Leftrightarrow x \succcurlyeq^{a} y$ for all $x, y \in A$. The independence conditions ROI-1 and ROI-2 imposed on this relation $R$ would then imply $x R y \Leftrightarrow x \succcurlyeq^{b} y$ for all $b, x, y \in A$. This is a slight strengthening of the characterization result, but it also makes the presentation less transparent.

The independence conditions ROI-1 and ROI-2 imply, essentially, that for any two reference outcomes $a$ and $b$ with $a$ preferred to $b$, the decision maker's preferences $\succcurlyeq^{a}$ and $\succcurlyeq^{b}$ coincide on the set lotteries involving only outcomes better than $a$, or only outcomes worse than $b$, or only outcomes between $a$ and $b$. This, in effect, produces the loss aversion coefficient $\lambda$ : the additional assumption on the probabilities in ROI- 2 guarantees that $\lambda$ is a constant. The condition ROD, finally, guarantees that $\lambda$ is nonnegative, so that we may truly speak of loss aversion.

We conclude with a comment on our assumption that reference outcomes are riskless. In Shalev's (2002) approach the reference outcome is not so much an alternative but rather a utility (or payoff) level. He considers bimatrix games; the two payoff matrices represent the basic utilities, and furthermore the players are loss averse with given loss aversion coefficients. A pair of strategies is a 'loss-aversion equilibrium' if there are payoff levels $r_{1}$ and $r_{2}$ such that in the game transformed according to (1) with $r_{1}$ and $r_{2}$ as reference levels, the pair of strategies under consideration is a Nash equilibrium resulting in the payoffs $r_{1}$ and $r_{2}$. Hence, the lottery induced by the strategy pair serves as (risky) reference outcome but the effect is the same as if there were a riskless outcome with $r_{1}$ and $r_{2}$ as payoffs: one could introduce an extra riskless outcome with $r_{1}$ and $r_{2}$ as basic utilities and nothing would change. Thus, despite our assumption of riskless reference outcomes Theorem 1 can still be used as a preference foundation for loss-aversion equilibrium. Köszegi and Rabin (2006) take an approach which is closely related to Shalev's, but then in the context of individual decision making. Their 'personal equilibrium' (PE) is the one-person pendant of loss-aversion equilibrium: indeed, "reformulating his [Shalev's] notion of lossaversion equilibrium using our utility function and applying it to individual decision-making corresponds to PE" (Köszegi and Rabin, 2006, p. 1144). 


\section{Appendix: proof of Theorem 1}

We first prove the implication (ii) $\Rightarrow$ (i). Assume that (ii) holds. Then, clearly, $R$ and $\succcurlyeq^{a}, a \in A$, are weak orderings.

To show COR, let $x, y, a \in A$. Then

$$
\begin{aligned}
x \succcurlyeq^{a} y \Leftrightarrow & u(x)-\lambda \cdot \max \{0, u(a)-u(x)\} \\
& \geqslant u(y)-\lambda \cdot \max \{0, u(a)-u(y)\} \\
\Leftrightarrow & u(x) \geqslant u(y) \\
& \Leftrightarrow \quad x R y
\end{aligned}
$$

where the first equivalence follows from (ii.2), the last from (ii.1), and the middle one by direct inspection.

EU is immediate from (ii.2). For ROI-1, let $a, b \in A$ with $a R b$ and $\ell, \ell^{\prime} \in \mathcal{L}$ with $a R x R b$ for all $x \in \operatorname{supp}(\ell) \cup \operatorname{supp}\left(\ell^{\prime}\right)$. Then

$$
\begin{aligned}
\ell \succcurlyeq^{a} \ell^{\prime} & \Leftrightarrow \sum_{x \in A} \ell(x)[u(x)-\lambda(u(a)-u(x))] \\
& \geqslant \sum_{x \in A} \ell^{\prime}(x)[u(x)-\lambda(u(a)-u(x))] \\
& \Leftrightarrow \sum_{x \in A} \ell(x) u(x) \geqslant \sum_{x \in A} \ell^{\prime}(x) u(x) \\
& \Leftrightarrow \quad \ell \succcurlyeq^{b} \ell^{\prime}
\end{aligned}
$$

implying ROI-1. For ROI-2, let $a, b \in A$ with $a R b$ and $\ell, \ell^{\prime} \in \mathcal{L}$ with $[\operatorname{supp}(\ell) \cup$ $\left.\operatorname{supp}\left(\ell^{\prime}\right)\right] \cap\{x \in A \mid a P x P b\}=\emptyset$ and $\ell(\{x \in A \mid b R x\})=\ell^{\prime}(\{x \in A \mid b R x\})$. Without loss of generality we assume $a P b$. Then

$$
\begin{aligned}
\ell \succcurlyeq^{a} \ell^{\prime} & \Leftrightarrow \sum_{x \in A: x R a} \ell(x) u(x)+\sum_{x \in A: b R x} \ell(x)[u(x)-\lambda(u(a)-u(x))] \\
& \geqslant \sum_{x \in A: x R a} \ell^{\prime}(x) u(x)+\sum_{x \in A: b R x} \ell^{\prime}(x)[u(x)-\lambda(u(a)-u(x))] \\
\Leftrightarrow & \sum_{x \in A: x R b} \ell(x) u(x)+\sum_{x \in A: b R x} \ell(x)[u(x)-\lambda(u(b)-u(x))] \\
& \geqslant \sum_{x \in A: x R b} \ell^{\prime}(x) u(x)+\sum_{x \in A: b R x} \ell^{\prime}(x)[u(x)-\lambda(u(b)-u(x))] \\
\Leftrightarrow & \ell \succcurlyeq^{b} \ell^{\prime}
\end{aligned}
$$

where for the second equivalence we have used that $\ell(\{x \in A \mid b R x\})=\ell^{\prime}(\{x \in$ $A \mid b R x\})$. This proves ROI- 2 .

Finally, to prove ROD, let $a, b \in A$ with $a R b$ and let $\ell \in \mathcal{L}$ with $\ell \succcurlyeq^{a} a$. Then

$$
E u^{b, \lambda}(\ell)=\sum_{x \in A: x R a} \ell(x) u(x)+\sum_{x \in A: a P x R b} \ell(x) u(x)
$$




$$
\begin{aligned}
& \quad+\sum_{x \in A: b P x} \ell(x)[u(x)-\lambda(u(b)-u(x))] \\
& \geqslant \sum_{x \in A: x R a} \ell(x) u(x) \\
& +\sum_{x \in A: a P x R b} \ell(x)[u(x)-\lambda(u(a)-u(x))] \\
& \quad+\sum_{x \in A: b P x} \ell(x)[u(x)-\lambda(u(a)-u(x))] \\
& =E u^{a, \lambda}(\ell)
\end{aligned}
$$

hence $E u^{b, \lambda}(\ell) \geqslant E u^{a, \lambda}(\ell) \geqslant E u^{a, \lambda}(a)=u(a)=E u^{b, \lambda}(a)$, implying $\ell \succcurlyeq^{b} a$. This proves ROD.

For the converse implication assume that (i) holds. In order to show (ii) we first construct $u$. Without loss of generality we assume that there are $s, t \in A$ with $t P s$. Fix an arbitrary representation $u^{s}$ of $\succcurlyeq^{s}$, hence $u^{s}(t)>u^{s}(s)$ by COR. Suppose that $x \in A, x R s$, and that there is a $y \in A$ with $y P x$. Then take the unique representation $u^{x}$ of $\succcurlyeq^{x}$ (as in EU) that satisfies $u^{x}(x)=u^{s}(x)$ and $u^{x}(y)=u^{s}(y)$. For $x \in A$ with $s P x$ we take the unique representation $u^{x}$ of $\succcurlyeq^{x}$ with $u^{x}(s)=u^{s}(s)$ and $u^{x}(t)=u^{s}(t)$. In case there exists a maximal outcome in $A$, i.e., an outcome $x$ such that $x R z$ for all $z \in A$, we simply take any arbirary representation $u^{x}$ of $\succcurlyeq^{x}$ with $u^{x}(x)=u^{s}(x)$. Altogether, we have fixed representations $u^{x}$ for all $x \in A$. Let $x, x^{\prime} \in A$ with $x R x^{\prime}$. By ROI-2 (with $a=x$ and $b=x^{\prime}$ ) we have $\ell \succcurlyeq^{x} \ell^{\prime} \Leftrightarrow \ell \succcurlyeq^{x^{\prime}} \ell^{\prime}$ for all $\ell, \ell^{\prime} \in \mathcal{L}$ with $z R x$ for all $z \in \operatorname{supp}(\ell) \cup \operatorname{supp}\left(\ell^{\prime}\right)$; by our choice of representations $u^{x}$ and $u^{x^{\prime}}$ this implies $u^{x}(z)=u^{x^{\prime}}(z)$ for all $z \in A$ with $z R x$, i.e., the functions $u^{x}$ and $u^{x^{\prime}}$ coincide for outcomes preferred to $x$ (and $x^{\prime}$ ) according to $R$. We now define $u$ by $u(x)=u^{x}(x)$ for all $x \in A$. By the preceding argument we have

$$
\text { for all } x, y \in A \text {, if } x R y \text { then } u(x)=u^{x}(x)=u^{y}(x) \text {. }
$$

We now check that $u$ satisfies (ii.1). Let $x, y \in A$. If $x R y$ then, by (2), $u(x)=u^{x}(x)=u^{y}(x) \geqslant u^{y}(y)$, where the inequality follows from COR. Hence, $u(x) \geqslant u(y)$. For the converse, let $u(x) \geqslant u(y)$ and suppose that $y P x$. Then by (2), $u(y)=u^{y}(y)=u^{x}(y)>u^{x}(x)$, where the inequality again follows from COR. So $u(y)>u^{x}(x)=u(x)$, a contradiction. Since $R$ is complete, it follows that $x R y$.

We proceed by defining the loss aversion coefficient. First, let $b \in A$ such that there are $a, c \in A$ with $a P b P c$. Let $\lambda^{b} \in \mathbb{R}$ be defined by the equation $u^{b}(c)=$ $u(c)-\lambda^{b}[u(b)-u(c)]$. Note that $u(b)=u^{b}(b)=u^{c}(b)$ by $(2)$ and that, by ROI-1 and EU, $u^{b}$ is a positive affine transformation of $u^{c}$ and thus of $u$ for all $x \in A$ with $b R x R c$. From this it is easily derived that $u^{b}(x)=u(x)-\lambda^{b}[u(b)-u(x)]$ for all $x \in A$ with $b R x R c$. Since $c$ was an arbitrary element of $A$ with $b P c$, we conclude that $u^{b}(x)=u(x)-\lambda^{b}[u(b)-u(x)]$ for all $x \in A$ with $b R x$.

We now show that $\lambda^{b}$, thus defined, is nonnegative. Let $0<p<1$ be defined by the equation $p u^{b}(c)+(1-p) u^{b}(a)=u^{b}(b)$, hence by $p u^{b}(c)+(1-p) u(a)=u(b)$ 
in view of (2); $p$ is well-defined since $u^{b}(a)>u^{b}(b)>u^{b}(c)$ by COR. Now ROD implies $p u^{c}(c)+(1-p) u^{c}(a) \geqslant u^{c}(b)$, hence $p u(c)+(1-p) u(a) \geqslant u(b)$ by $(2)$. Thus, we conclude that $u(c) \geqslant u^{b}(c)$; since $u(b)>u(c)$ (by (ii.1)) and since (by definition) $u^{b}(c)=u(c)-\lambda^{b}[u(b)-u(c)]$, this implies $\lambda^{b} \geqslant 0$.

Next, suppose we apply this construction to two different points $b, b^{\prime} \in A$ with $a P b P b^{\prime} P c$, resulting in $\lambda^{b}, \lambda^{b^{\prime}} \geqslant 0$. We will show that $\lambda^{b}=\lambda^{b^{\prime}}$. Consider a lottery $\ell$ assigning probabilities $\alpha, \frac{1}{2}-\alpha, \frac{1}{2}-\beta, \beta$ and a lottery $\ell^{\prime}$ assigning probabilities $\alpha^{\prime}, \frac{1}{2}-\alpha^{\prime}, \frac{1}{2}-\beta^{\prime}, \beta^{\prime}$ to $c, b^{\prime}, b, a$, respectively, where $0<\alpha<\alpha^{\prime}<1$ and $0<\beta<\beta^{\prime}<1$ are such that $u^{b}(\ell)=u^{b}\left(\ell^{\prime}\right)$, i.e.

$$
\begin{aligned}
u^{b}(\ell)= & \alpha\left(u(c)-\lambda^{b}[u(b)-u(c)]\right)+\left(\frac{1}{2}-\alpha\right)\left(u\left(b^{\prime}\right)-\lambda^{b}\left[u(b)-u\left(b^{\prime}\right)\right]\right) \\
& +\left(\frac{1}{2}-\beta\right) u(b)+\beta u(a) \\
= & \alpha^{\prime}\left(u(c)-\lambda^{b}[u(b)-u(c)]\right)+\left(\frac{1}{2}-\alpha^{\prime}\right)\left(u\left(b^{\prime}\right)-\lambda^{b}\left[u(b)-u\left(b^{\prime}\right)\right]\right) \\
& +\left(\frac{1}{2}-\beta^{\prime}\right) u(b)+\beta^{\prime} u(a) \\
= & u^{b}\left(\ell^{\prime}\right) .
\end{aligned}
$$

(It is not difficult to see that such lotteries exist.) The second equation can be simplified to

$$
\left(1+\lambda^{b}\right)\left(\alpha^{\prime}-\alpha\right)\left(u\left(b^{\prime}\right)-u(c)\right)=\left(\beta^{\prime}-\beta\right)(u(a)-u(b)) .
$$

By ROI-2, $u^{b}(\ell)=u^{b}\left(\ell^{\prime}\right)$ implies $u^{b^{\prime}}(\ell)=u^{b^{\prime}}\left(\ell^{\prime}\right)$. Writing out and then simplifying the latter equality yields, similarly,

$$
\left(1+\lambda^{b^{\prime}}\right)\left(\alpha^{\prime}-\alpha\right)\left(u\left(b^{\prime}\right)-u(c)\right)=\left(\beta^{\prime}-\beta\right)(u(a)-u(b)) .
$$

It follows that $\lambda^{b}=\lambda^{b^{\prime}}$, and we write $\lambda$ for this common value.

If $b \in A$ is such that either $x R b$ for all $x \in A$ or $b R x$ for all $x \in A$ then we can simply take $\lambda^{b}=\lambda$. This concludes the construction of the loss aversion coefficient $\lambda$, and clearly (ii.2) is satisfied. The final claim - uniqueness of $u$ up to a positive affine transformation - follows since by construction (or, equivalently, by (ii.2)) $u(x)=u^{a}(x)$ for all $a \in A$ and $x \in A$ with $x R a$, and each $u^{a}$ is unique up to a positive affine transformation.

\section{References}

Abdellaoui M, Bleichrodt H, Paraschiv C (2007) Loss aversion under prospect theory: a parameter-free measurement. Management Science 53:16591674

Bleichrodt H (2007) Reference-dependent utility with shifting reference points and incomplete preferences. Journal of Mathematical Psychology 51:266276 
Dittmann I, Maug E, Spalt O (2009) Sticks or carrots? Optimal CEO compensation when managers are loss averse. Journal of Finance, forthcoming

Driesen B, Perea A, Peters H (2010) On loss aversion in bimatrix games. Theory and Decision 68:367-391

Dunn LF (1996) Loss aversion and adaptation in the labour market: empirical indifference functions and labour supply. Review of Economics and Statistics 78:441-450

Freund C, Özden C (2008) Trade policy and loss aversion. American Economic Review 98:1675-1691

Genesove D, Mayer C (2001) Loss aversion and seller behavior: evidence from the housing market. Quarterly Journal of Economics 116:1233-1260

Götte L, Huffman D, Fehr E (2004) Loss aversion and labor supply. Journal of the European Economic Association 2:216-228

Herstein IN, Milnor J (1953) An axiomatic approach to measurable utility. Econometrica 21:291-297

Kahneman D (2003) Experiences of collaborative research. American Psychologist 58:723-730

Kahneman D, Tversky A (1979) Prospect theory: an analysis of choice under risk. Econometrica 47:263-291

Köbberling V, Wakker PP (2005) An index of loss aversion. Journal of Economic Theory 122:119-131

Köszegi B, Rabin M (2006) A model of reference-dependent preferences. Quarterly Journal of Economics 121:1133-1165

Schmidt U (2003) Reference dependence in cumulative prospect theory. Journal of Mathematical Psychology 47:122-131

Rosenblatt-Wisch R (2008) Loss aversion in aggregate macroeconomic time series. European Economic Review 52:1140-1159

Shalev J (2002) Loss aversion equilibrium. International Journal of Game Theory 29:269-287

Sugden R (2003) Reference-dependent subjective expected utility. Journal of Economic Theory 111:172-191

Tversky A, Kahneman D (1991) Loss aversion in riskless choice: a referencedependent model. Quarterly Journal of Economics 56:1039-1061

Tversky A, Kahneman D (1992) Advances in prospect theory: cumulative representation of uncertainty. Journal of Risk and Uncertainty 5:297-323 